\title{
Optimization for cutting force and material removal rate in milling thin-walled parts
}

\author{
Sheng $\mathrm{Qu}^{1,2, a}$ and Mingqin Zhang ${ }^{1, b}$ \\ ${ }^{1}$ School of Mechanical Engineering, Shandong Jianzhu University, Jinan 250101, China; \\ ${ }^{2}$ University of Chinese Academy of Sciences, Beijing 100049, China. \\ aqsqdlg@163.com, ${ }^{b} 651073314 @ q q . c o m$
}

Keywords: Thin-walled parts Machining parameters Optimization NSGA-II

\begin{abstract}
Conservative milling parameters are usually adopted in actual milling. This paper presents an optimization procedure to determine the optimum combinations of machining parameters for minimal cutting force and maximal machining efficiency. The regression model for cutting force is developed as objective function according to experimental results. The objectives under investigation in this study are cutting force and material removal rate subjected to constraints conditions. A non-dominated sorting genetic algorithm (NSGA-II) is then adopted to solve this multi-objective optimization problem. The optimized combinations of machining parameters are achieved by the Pareto optimal solutions.
\end{abstract}

\section{Introduction}

Large numbers of components used in aerospace field are designed as thin-walled structures owing to their physical performances, such as less weight and more structure strength. However, deformation is easy to appear because of their low rigidity in machining process. Cutting parameters optimization is performed to determine optimal milling parameters to satisfy various industrial demands.

Initially, efforts are focused on optimizing parameters for one single objective (e.g., cutting forces, tool wear, and machining efficiency). Oktem et al. [1] resorted to genetic algorithm and neural networks to get the optimal parameters for surface roughness in milling mold faces. Vijayakumar et al. [2] obtained the multipass turning parameters to minimizing the unit production cost by means of ant colony system. Natarajan et al. [3] determined optimal parameters for longer tool life by genetic algorithm and particle swarm method. However, due to the complex nature of the machining processes, often optimization problems have several contradictory objectives to be optimized simultaneously. That optimizing one objective causes the other objectives to be poor makes the selection of optimal parameters become difficult. Multi-objective optimization methodologies have demonstrated their usefulness in finding a well-distributed Pareto optimal solutions. Yang and Natarajan [4] performed simultaneous optimization of tool wear and material removal rate to obtain optimal machining parameters. Govindan and Joshi [5] investigated the effects of milling parameters in order to maximize milling efficiency and minimize tool wear rate. .

This paper focuses on optimizing machining parameters in milling thin-walled parts based on NSGA-II. Simultaneous optimization of cutting force and material removal rate in thin-walled parts machining process are carried out in this study.

\section{Experimental procedure}

In this work, a series of cutting tests of milling thin-walled plates have been conducted to develop statistical models of cutting force and surface roughness by means of regression analysis. These models are applied in the procedure of searching for optimal machining parameters.

The parts are machined on ME650 three-dimensional vertical machining center. The cutter used in experiments is a four-flute flat-end milling cutter (diameter $16 \mathrm{~mm}$ and helix angle 30 degrees), which is made of solid carbide and coated with TiSiN. A rectangular thin-walled plate with size of 
$120 \times 100 \times 6 \mathrm{~mm}$ is selected as the workpiece. The workpiece selected for the experiments is die steel NAK80. A Kistler 9123C dynamometer is used to measure the cutting force, as shown in Fig. 1. The cutting forces are measured at each milling test.

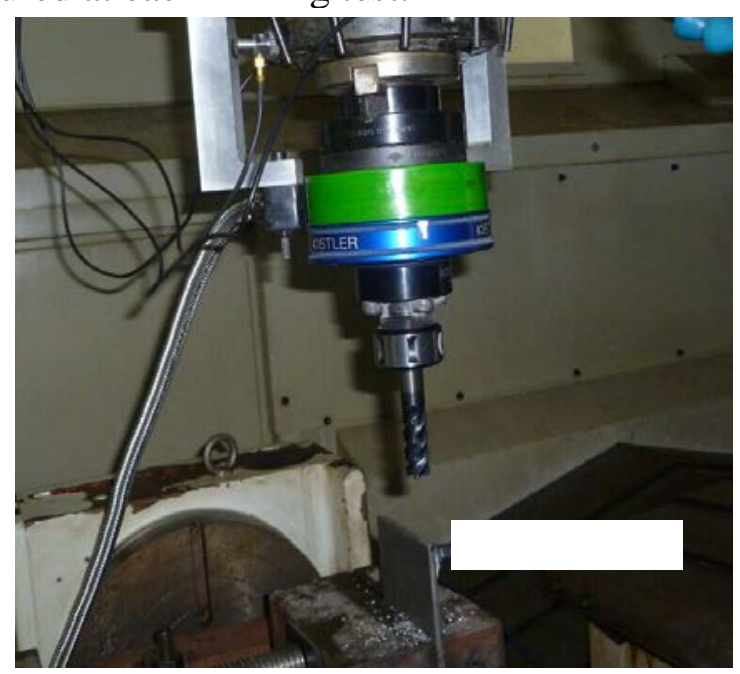

Fig. 1 Milling experiment setup

Table 1 Experimental conditions and results

\begin{tabular}{cccccc}
\hline No. & \multicolumn{3}{c}{ Experimental conditions } & \multicolumn{2}{c}{ Experimental results } \\
\cline { 2 - 6 } & $\begin{array}{c}\text { Spindle speed } \\
(n, \mathrm{r} / \mathrm{min})\end{array}$ & $\begin{array}{c}\text { Feed per tooth } \\
(f, \mathrm{~mm})\end{array}$ & $\begin{array}{c}\text { Axial depth } \\
\left(a_{p}, \mathrm{~mm}\right)\end{array}$ & $\begin{array}{c}\text { Cutting force } \\
(\mathrm{Fa}, \mathrm{N})\end{array}$ & $\begin{array}{c}\text { Removal rate } \\
\left(\mathrm{M}, \mathrm{mm}^{3} / \mathrm{min}\right)\end{array}$ \\
\hline 1 & 1600 & 0.15 & 0.4 & 27.3 & 384 \\
2 & 1600 & 0.15 & 0.55 & 42.7 & 503 \\
3 & 1600 & 0.15 & 0.7 & 58.5 & 672 \\
4 & 1600 & 0.2 & 0.4 & 33.2 & 512 \\
5 & 1600 & 0.2 & 0.55 & 52.8 & 704 \\
6 & 1600 & 0.2 & 0.7 & 70.2 & 896 \\
7 & 1600 & 0.25 & 0.4 & 39.2 & 640 \\
8 & 1600 & 0.25 & 0.55 & 54.6 & 880 \\
9 & 1600 & 0.25 & 0.7 & 69.8 & 1120 \\
10 & 2100 & 0.15 & 0.4 & 35.3 & 504 \\
11 & 2100 & 0.15 & 0.55 & 49.6 & 693 \\
12 & 2100 & 0.15 & 0.7 & 68.5 & 882 \\
13 & 2100 & 0.2 & 0.4 & 39.7 & 672 \\
14 & 2100 & 0.2 & 0.55 & 56.5 & 924 \\
15 & 2100 & 0.2 & 0.7 & 73.4 & 1176 \\
16 & 2100 & 0.25 & 0.4 & 43.9 & 840 \\
17 & 2100 & 0.25 & 0.55 & 58.3 & 1155 \\
18 & 2100 & 0.25 & 0.7 & 73.6 & 1470 \\
19 & 2600 & 0.15 & 0.4 & 38.5 & 624 \\
20 & 2600 & 0.15 & 0.55 & 52.7 & 858 \\
21 & 2600 & 0.15 & 0.7 & 72.3 & 1092 \\
22 & 2600 & 0.2 & 0.4 & 43.6 & 832 \\
23 & 2600 & 0.2 & 0.55 & 61.3 & 1144 \\
24 & 2600 & 0.2 & 0.7 & 82.3 & 1456 \\
25 & 2600 & 0.25 & 0.4 & 48.9 & 1040 \\
26 & 2600 & 0.25 & 0.55 & 71.4 & 1430 \\
27 & 2600 & 0.25 & 0.7 & 85.6 & 1820 \\
\hline
\end{tabular}

The milling process involves various milling parameters such as spindle speed, feed per tooth, and axial depth of cut. In the process of milling thin-walled part, the radial depth of cut ae is fixed to $1 \mathrm{~mm}$ in this study. The tested parameters including spindle speed $n$, feed per tooth $\mathrm{f}$, and axial depth of cut ap are employed to build experimental tables. The three parameters with every three levels constitute the L 27 orthogonal array, as shown in Table 1. It also lists experimental results of the cutting force Fa 
and material removal rate $M$. In this table, the cutting force Fa refers to the mean value in each milling process.

\section{Statistical model for optimization}

Regression analysis is a method for investigating functional relationships between input and output parameters. In this study, input parameters include spindle speed, feed per tooth, and axial depth of cut. The experimental results of cutting force and surface roughness are applied to establish statistical model by regression analysis method. The regression equations between cutting force and input parameters can be modeled based on second-order polynomial equation as given in the following:

$$
Y=\alpha_{0}+\sum_{i=1}^{k} \alpha_{i} X_{i}+\sum_{i \leq j}^{k} \alpha_{i} X_{i} X_{j}
$$

where $\alpha_{i}$ represents the coefficient and $X_{i}$ is the input parameter. All the unknown coefficients are determined using regression with the aid of Matlab software.

The cutting force model based on regression method is developed according to the measured results in Table 1 and the model is given as follows:

$$
\begin{aligned}
F a & =-52.07-0.006 n+381.01 f+80.35 a_{p}+0.0243 n \cdot f \\
& -13.333 f \cdot a_{p}+0.0116 n \cdot a_{p}-784.44 f^{2}+9.8765 a_{p}{ }^{2}
\end{aligned}
$$

The acquired cutting force model cannot be directly applied in the process of analysis and estimation. It is necessary to further confirm the statistical regularity of the model. The regression equation needs to be verified by the normality assumption [6]. F calibration is adopted to test the reliability of the regression equation. The $\mathrm{R}^{2}$ value of the second-order polynomial model for cutting force is $98.64 \%$, which is close to 1 . It shows that the regression equation is highly significant at a 95\% confidence value.

Material removal rate needs to be considered when determining the machining parameters because the higher efficiency is of great significance in such operations. Material removal rate is defined as the volume of material that is removed from the part per unit time and it is a function of the milling parameters, which is expressed as:

$$
M=n \cdot f \cdot Z \cdot a_{p} \cdot a_{e}
$$

where $\mathrm{Z}$ is the tooth number.

\section{Results of multi-objective optimization}

Enhanced from single objective optimization genetic algorithm, many multi-objective optimization approaches are proposed. Among these approaches, NSGA-II is most widely used to optimize the machining parameters. Using NSGA-II, a set of optimal solutions called Pareto optimal fronts would be obtained that in general any of these solutions has no predominance to another [7].

In production process, the major goal is to fabricate high quality thin-walled parts with high efficiency. That is, the main problem is to obtain the minimum deformation and best milling efficiency concerning the industrial demands. Thus, the minimal cutting force and the maximal material removal rate are optimization objectives. The objective functions are given as follows:

$$
\left\{\begin{array}{l}
\text { Objective }=\min [\mathrm{Fa}, 1 / \mathrm{M}] \\
\text { s.t. }=\left\{\begin{array}{l}
n=1000 \sim 3000 \mathrm{r} / \mathrm{min} \\
f=0.1 \sim 0.3 \mathrm{~mm} \\
a_{p}=0.3 \sim 1 \mathrm{~mm}
\end{array}\right.
\end{array}\right.
$$

In the optimization procedure, the population size is taken as 200 and the evolutionary generation is adopted as 50. After optimization, the Pareto-optimal front obtained by NSGA-II is in Fig. 4.

All the points in Fig. 4 denote an optimal combination of machining parameters that has no predominance to each other. The choice of optimum combination milling parameters over the other 
depends on the desired machining criterion of the thin-walled part. As it is depicted in Fig. 4, it is clear that material removal rate $M$ value increases with the increase of cutting force $F a$ value. In addition, it can be seen from Fig. 4 that the solutions in region A can get optimal cutting force and material removal rate.

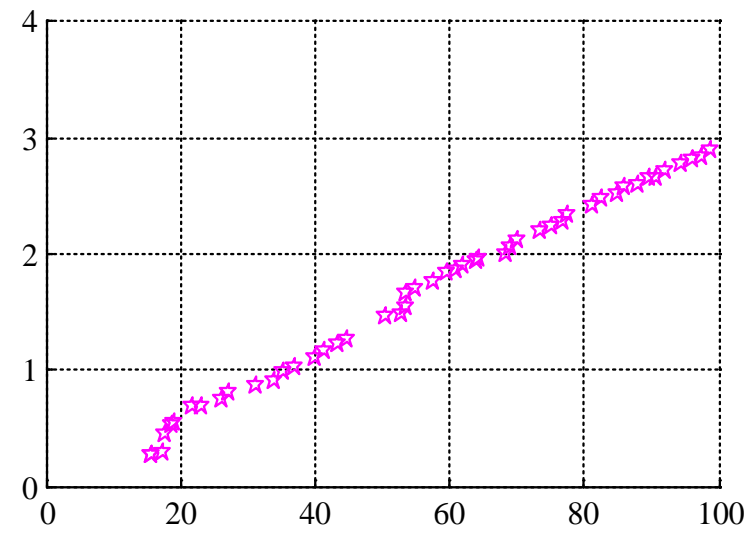

Fig. 4 Pareto optimal front for optimization objectives

An experimental confirmation test is conducted to demonstrate the effectiveness the optimization method. The experimental results are shown in Table 2. Based on the conservative parameters, the cutting force is small to reduce deformation and the value of cutting force is $47.6 \mathrm{~N}$. After optimization, the value of cutting force is $43.9 \mathrm{~N}$, very close to 47.6 . But the material removal rate increases obviously, and it is increased by $78 \%$ compared with that before optimization. Hence, the optimized machined parameters show good reliability to guarantee the quality and improve milling efficiency.

Table 2 Comparison of milling parameters and experimental results

\begin{tabular}{cccccc}
\hline & $n, \mathrm{r} / \mathrm{min}$ & $f, \mathrm{~mm}$ & $a_{p}, \mathrm{~mm}$ & $F a, \mathrm{~N}$ & $M, \mathrm{~mm}^{3} / \mathrm{min}$ \\
\hline $\begin{array}{c}\text { Conservative } \\
\text { parameters }\end{array}$ & 2000 & 0.15 & 0.55 & 47.6 & 660 \\
optimization & 2832 & 0.24 & 0.428 & 43.9 & 1175 \\
\hline
\end{tabular}

\section{Conclusions}

In this paper, an attempt has been made to solve multi-objective optimization problem in milling thin-walled plates by using NSGA-II. The conflicting objectives, namely, cutting force and material removal rate, have been considered for optimization simultaneously. As a consequence, the optimum combinations of machining parameters and output objectives are acquired. The proposed method provides an applicable range wide range of solutions for decision maker, which helps to guarantee the high quality of the machined thin-walled parts as well as improve milling efficiency.

\section{References}

[1] H. Oktem, F. Erzurumlu, F. Erzincanli, Prediction of minimum surface roughness in end milling mold parts using neural network and genetic algorithm, Mater Design. 27 (2006) 735-744.

[2] K. Vijayakumar, G. Prabhaharan, P. Asokan, P. Saravanan, Optimization of multi-pass turning operations using ant colony system, Int J Mach Tools Manuf. 43 (2003) 1633-1639.

[3] U. Natarajan, V.M. Periasamy, R. Saravanan, Application of particle swarm optimization in artificial neural network for the prediction of tool life, Int J Adv Manuf Technol. 28 (2006) 1084-1088.

[4] S.H. Yang, U. Natarajan, Multi-objective optimization of cut-ting parameters in turning process using differential evolution and non-dominated sorting genetic algorithm-II approaches, Int J Adv Manuf Technol. 49 (2010) 773-784. 
[5] P. Govindan, S.S. Joshi, Experimental characterization of material removal in dry electrical discharge drilling, Int J Mach Tools Manuf. 50 (2010) 431-44.

[6] X. Chen, L. Ma, C. Li, X. Cao, Experimental study and genetic algorithm-based optimization of cutting parameters in cutting engineering ceramics, Int J Adv Manuf Technol. 74 (2014) 807-817.

[7] K. Deb, A. Pratap, S. Agarwal, T. Meyarivan, A fast and elitist multiobjective genetic algorithm: NSGA- II, IEEE Trans Evol Comput. 6 (2002) 182-197. 